\section{Binocular rivalry of real vs. subjective contours}

\author{
DRAKE R. BRADLEY \\ Bates College, Lewiston, Maine
}

Previous research has established that subjective contours have many of the same functional consequences for vision as real contours. Various studies have shown that subjective contours can undergo and/or induce illusory distortions, visual masking, apparent movement, perceptual fragmentation, perceptual ambiguity, three-dimensional reversibility, shape distortions during rotation, and tilt, color, motion, and figural aftereffects (Bradley \& Dumais, 1975; Bradley \& Lee, 1982; Bradley \& Petry, 1977; Farné, 1968; Goldstein \& Weintraub, 1972; Gregory, 1972; Halpern \& Warm, 1980; Kanizsa, 1974; Pastore, 1971; Smith \& Over, 1975, 1976; Stadler \& Dieker, 1969; von Grünau, 1979; Weisstein, Maguire, \& Berbaum, 1977; Weisstein, Matthews, \& Berbaum, 1974). While a number of studies have investigated the stereoscopic production, enhancement, or erasure of subjective contours (Gregory \& Harris, 1974; Harris \& Gregory, 1973; Julesz \& Frisby, 1975; Lawson, Cowan, Gibbs, \& Whitmore, 1974; Whitmore, Lawson, \& Korzora, 1976), apparently no one has investigated whether dichoptic presentation of intersecting subjective contours results in binocular rivalry and contralateral suppression of the contours. The purpose of this note is to present two demonstrations which provide a preliminary answer to this question.

Figure $1 \mathrm{~A}$ shows an upright and an inverted black outline triangle, each superimposed on three black disks. The six black lines converging on each triangle and the dot in the center are provided to facilitate proper alignment of the two triangles when viewed dichoptically. When these triangles are presented in the left and right channels of a stereoscope, binocular rivalry is observed where the contours of the two outline triangles intersect in the combined view. Zones of contralateral suppression are seen surrounding the contours that are dominant at any given moment. These zones mask the nondominant contour in the immediate vicinity of the dominant contour. One of the two triangles may disappear entirely, along with the three black disks, only to reappear later, displacing the previously dominant triangle. When both triangles are visible at the same time, the contours of one generally suppress those of the other at all six intersection points. However, sometimes the contours of one triangle suppress those of the other only at some of the intersection points (e.g., the top corner of the upright triangle is dominant, but the

The author's mailing address is: Bates College, Department of Psychology, Lewiston, Maine 04240.
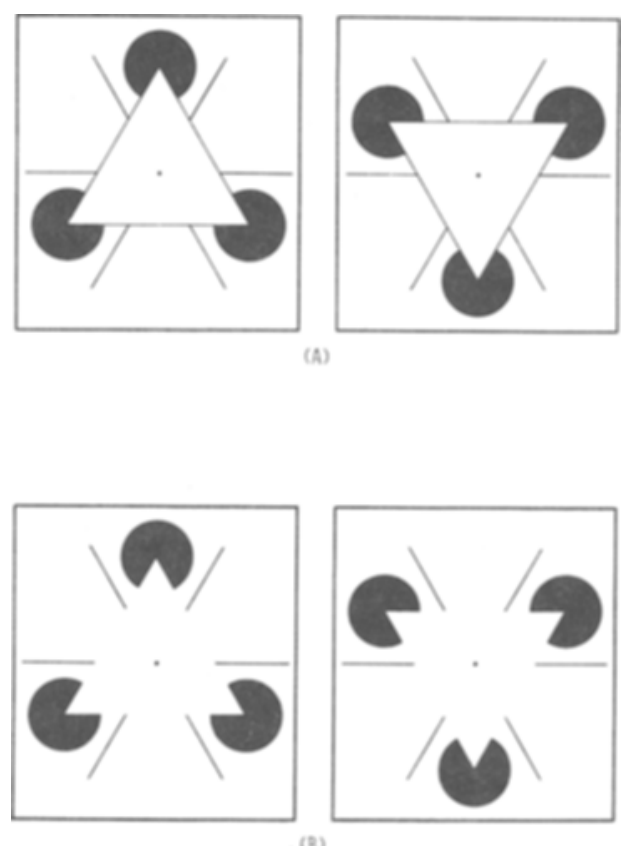

Figure 1. Stimuli used for assessing binocular rivalry of real (A) and subjective (B) contours.

bottom two corners are not). This creates the impression of two "interleaved" triangles.

If the production of subjective contours involves mechanisms similar to those of real contours, then the effects described above might also be observed during the dichoptic viewing of intersecting subjective contours. Figure 1B is identical to Figure 1A, except that the real contours outlining the triangles have been removed. When viewed individually, the left and right panels of Figure 1B result in the perception of an upright and inverted subjective triangle, respectively. In the combined view, the subjective contours of the two triangles will "intersect" in six different locations, just as for the real contour triangles of Figure 1A. These points of intersection represent potential sites for binocular rivalry and contralateral suppression. The fact that the subjective contours of Figure $1 \mathrm{~B}$ and the real contours of Figure $1 \mathrm{~A}$ are not equivalent in one important respect is considered below. For the moment, we are simply concerned with determining whether or not subjective contours manifest rivalry and suppression effects analogous to those observed with real contours.

When Figure $1 B$ is viewed in a stereoscope, a very interesting effect is observed: instead of showing rivalry, the monocular subjective contours merge to form the interior corners of a six-pointed star. The star represents a new subjective contour object generated by the binocular integration of the individual subjective 
triangles. This integration is not a simple summation or superimposition of the two sets of subjective contours. Instead, selective portions of the monocular subjective contours are blended together to produce a new subjective contour object. Specifically, the corners of each monocular triangle are combined to form the corners of the six-pointed star. The "center" portions of the subjective contours forming the sides of the triangles are not utilized in the formation of the six-pointed star. This selective use of contour information from the monocular views to produce a new object is not observed in Figure 1A. In those instances in which the observer can see both triangles simultaneously, the center portions of the sides of the triangles form a hexagonal shape in the center of the combined view.

While the combined view of the triangles in Figure 1A can result in the impression of an outline star, it is unstable because the intersection points show rivalry and suppression of the contours. In contrast, the subjective star generated by Figure IB is quite stable. The subjective contours that form the interior corners of the star appear as single contours which change direction, and not as separate contours which converge, pass through each other, and rival each other. However, this perception of a six-pointed star is sometimes replaced by the perception of two triangles, one superimposed on the other. Unlike Figure 1A, the perception of a triangle (rather than a star) is not associated with the suppression of the other triangle: instead, one triangle is seen on top of the other, and the subjective contours of the latter are seen to extend right up to the "edges" of the former, and to disappear underneath it. In this case, the "center" portions of the monocular subjective contours of the "top" triangle are utilized in the combined view, whereas those of the "bottom" triangle are not. With prolonged inspection, the relative positions of the triangles may reverse, or they may once again merge into a six-pointed star.

The alternations in the organization of Figure 1B are not the result of binocular rivalry, because they also occur when viewing (nondichoptically) an actual sixpointed star (Bradley \& Dumais, 1975). Rather, they would seem to result from the inherent perceptual ambiguity of the star configuration itself, which allows for several possible organizations of the subjective contours. Conversely, the fluctuating appearance of the forms in Figure 1A result primarily from the binocular rivalry of the contours, and/or the complete suppres. sion of one monocular input by the other. The reader can best verify these observations by Xeroxing Figures $1 \mathrm{~A}$ and $\mathrm{IB}$ and viewing them in a stereoscope, or by viewing Figures $1 \mathrm{~A}$ and $\mathrm{B}$ with eyes converged at a point which brings the left and right panels of the figure in to alignment.

Unfortunately, comparison of binocular rivalry in Figures $1 \mathrm{~A}$ and $1 \mathrm{~B}$ is partly confounded. The lines demarcating the real contour triangles of Figure 1A represent two-step brightness gradients (light-darklight), whereas the subjective contours bounding the triangles of Figure 1B represent onc-step brightness gradients. The latter is duc to the fact that subjective contours are normally associated with discrete changes in apparent brightness, with the subjective con tour object appearing brighter (lighter) than its background. Perhaps only those contours arising from a two-step brightness gradient manifest rivalry and contralateral suppression. To assess this possibility, a third figure was prepared by cutting out two triangles and superimposing them on the two subjective triangles of Figure 1B. In an attempt to simulate the apparent brightness difference experienced with subjective contour figures, the reflectance values of the superimposed triangles and the background were somewhat different $(84.2 \%$ vs. 73.4\%).

When the modified version of Figure $1 \mathrm{~B}$ is viewed in a stereoscope, binocular rivalry and contralateral suppression are observed at the intersection points of the one-step contours. The rivalry is not as prevalent as that seen in Figure 1A, and it is somewhat easier to maintain the impression of a six-pointed star. However, the star does not have the uniform appearance of the star created by the merging of the subjective triangles of Figure 1B. The center portions of the sides of the triangles are still clearly visible, and form a hexagonal shape in the center of the combined view (as in Figure 1A). Hence, binocular viewing of intersecting one-step contours does not result in the same effects as observed for the subjective contours of Figure 1B.

In conclusion, the observations presented here suggest that subjective contours may follow laws of binocular interaction different from those of real (two-step or one-step) contours. Dichoptic presentation of subjective contours differing in orientation does not result in binocular rivalry or contralateral suppression of the contours. Instead, the subjective contours merge to form a new subjective contour object in the combined view. This object may, in turn, be replaced by other stable arrangements of subjective contours representing different utilizations, in part or whole, of the individual subjective contours from the two monocular views. These findings imply that the binocular integration of subjective contours is inherently more flexible than that of real contours. Consequently, theories stressing a fundamental similarity in the processing of real and subjective contours should take this fact into account.

\section{REFERENCES}

Bradley, D. R., \& Dumals, S. T. Ambiguous cognitive contours. Nature, 1975, 257, 582-584.

Bradlex, D. R., \& Lee, K. Animated subjective contours. Perception \& Psychophysics, 1982, in press.

Bradley, D. R., \& Petry, H. M. Organizational determinants of subjective contour: The subjective Necker cube. American Journal of Psychology, 1977, 90, 253-262. 
FARNÉ, M. Alcune osservazioni con linee virtuali e margini quasi percettivi. Bolletino Società Italiana Biologia Sperimentale, $1968,44,1613-1616$.

Goldstein, M. B., \& Weintraub, D. J. The parallel-less Poggendorff: Virtual contours put the illusion down but not out. Perception \& Psychophysics, 1972, 11, 353-355.

Gregony, R. L. Cognitive contours. Nature, 1972, 238, 51 -52.

Gregory, R. L., \& Harris, J. P. Illusory contours and stereo depth. Perception \& Psychophysics, 1974, 15, 411-416.

HALPERN, D. F., \& WARM, J. S. The disappearance of real and subjective contours. Perception \& Psychophysics, 1980, 28, 229-235.

Harris, J. P., \& Gregory, R. L. Fusion and rivalry of illusory contours. Perception, 1973, 2, 235-247.

Julesz, B., \& Frisby, J. B. Some new subjective contours in random-line stereograms. Perception, 1975, 4, 145-150.

KanizsA, G. Contours without gradients or cognitive contours? Italian Journal of Psychology, 1974, 1, 93-113.

Lawson, R. B., Cowan, E., Gibbs, T. D., \& Whitmore, C. G. Stereoscopic enhancement and erasure of subjective contours. Journal of Experimental Psychology, 1974, 103, 1142-1146.

Pastore, N. Selective history of theories of visual perception 1650-1950. New York: Oxford University Press, 1971.
Smith, A., \& Oven, R. Tilt aftereffects with subjective contours. Nature, 1975, 257, 581-582.

Smith, A. T., \& Over, R. Color-selective tilt aftereffects with subjective contours. Perception \& Psychophysics, 1976, 20, 305-308.

Stader, M., \& Die ker, J. Margini quasi-percettivi aftereffects figurali. Rivista di Psicologia, 1969, 63, 95-104.

von Grunau, M. W. The involvement of illusory contours in stroboscopic motion. Perception \& Psychophysics, 1979, 25, 205-208.

Weisstein, N., Maguire, W., \& Beabaum, K. A phantommotion aftereffect. Science, 1977, 198, 955-958.

Weisstein, N., Matthews, K., \& Berbaum, K. Illusory contours can mask real contours. Bulletin of the Psychonomic Society, 1974, 4, 266.

Whitmore, C. L. G., Lawson, R. B., \& Kozora, C. E. Subjective contours in stereoscopic space. Perception \& Psychophysics, 1976, 19, 211-213.

(Manuscript received April 16, 1982; accepted for publication April 26, 1982.)

\section{Twenty-Third Annual Meeting of the Psychonomic Society}

The twenty-third annual meeting of the Psychonomic Society will be held at the Hyatt Regency Hotel in Minneapolis, November 11-13, 1982. The call for papers was mailed to Society members during May 1982 . The deadline for the receipt of abstracts was July 1 , 1982. Papers dealing with the application of psychological science to real-world problems were encouraged. The program and hotel reservation forms will be mailed to members in the early fall. Also, the program will be printed in the September issue of the Bulletin of the Psychonomic Society. The Secretary-Treasurer, J. Bruce Overmier, at the Department of Psychology, University of Minnesota, Minneapolis, Minnesota 55455 [(612) 373-3430] may be contacted for further information. 